\title{
ZEROS IN LINEAR SYSTEMS WITH TIME DELAY IN STATE
}

\author{
JERZY TOKARZEWSKI \\ Faculty of Electrical Engineering \\ Warsaw University of Technology, Pl. Politechniki 1, 00-661 Warsaw, Poland \\ e-mail: jtokarzewski@zkue.ime.pw.edu.pl
}

\begin{abstract}
The concept of invariant zeros in a linear time-invariant system with state delay is considered. In the state-space framework, invariant zeros are treated as triples: complex number, nonzero state-zero direction, input-zero direction. Such a treatment is strictly related to the output-zeroing problem and in that spirit the zeros can be easily interpreted. The problem of zeroing the system output is discussed. For systems of uniform rank, the first nonzero Markov parameter comprises a certain amount of information concerning invariant zeros, output-zeroing inputs and zero dynamics. General formulas for output-zeroing inputs and zero dynamics are provided.
\end{abstract}

Keywords: linear systems, time delay in state, state-space methods, output-zeroing problem, invariant zeros.

\section{Introduction}

The problem of zeroing the output of a standard linear system $\mathrm{S}(\mathrm{A}, \mathrm{B}, \mathrm{C})$ is, as is known (Isidori, 1995; MacFarlane and Karcanias, 1976; Tokarzewski, 2002; 2006), strictly connected with the notion of the zeros of the system. These zeros are defined in many, not necessarily equivalent, ways. For a survey of these definitions, see (MacFarlane and Karcanias, 1976; Schrader and Sain, 1989; Tokarzewski, 2002; 2006). The most commonly used definition of zeros employs the Smith canonical form of the system matrix and determines these (Smith) zeros as the roots of diagonal (invariant) polynomials of the Smith form. Equivalently, Smith zeros are defined as the points of the complex plane where the rank of the system matrix drops below its normal rank. Another group of definitions employs the module-theoretic setting (Bourles and Fliess, 1997; Schrader and Sain, 1989).

All the above mentioned definitions consider zeros merely as complex numbers and for this reason may create certain difficulties in their dynamical interpretation. MacFarlane and Karcanias (1989), added to the notion of Smith zeros the notions of state-zero and input-zero directions and formulated the so-called output-zeroing problem. Another definition of zeros (called invariant), employing the system matrix and zero directions, was used in (Tokarzewski, 2002; 2006). These zeros are treated there as triples: complex number, non-zero state-zero direction, and input-zero direction and are defined as fol- lows. A complex number $\lambda$ is an invariant zero of a system $S(A, B, C)$, where $A, B, C$ are real matrices of dimensions $n \times n, n \times m$ and $r \times n$, respectively, if there exist vectors $0 \neq x^{o} \in \mathbb{C}^{n}$ and $g \in \mathbb{C}^{m}$ such that

$$
P(\lambda)\left[\begin{array}{c}
x^{o} \\
g
\end{array}\right]=\left[\begin{array}{l}
0 \\
0
\end{array}\right],
$$

where

$$
P(s)=\left[\begin{array}{cc}
s I-A & -B \\
C & 0
\end{array}\right]
$$

denotes the system matrix for $S(A, B, C)$. Invariant zeros constitute an extension of the notion of Smith zeros. The latter are involved in several problems of linear control systems, such as zeroing the output, tracking the reference output, disturbance decoupling, noninteracting control or output regulation (Isidori, 1995; Marro, 1996; Sontag, 1990).

Unfortunately, for systems with delays the concept of invariant zeros is not extensively discussed in the relevant literature (Pandolfi, 1982; 1986).

The paper is organized as follows: A system $S\left(A, A_{1} e^{-s h}, B, C\right)$ of the form (1) below is discussed. We introduce first the concept of invariant zeros. In Section 3, a dynamical interpretation of those zeros is given. We show also that for an asymptotically stable system (1) an output-zeroing input (if such inputs exist), when applied to the system under an arbitrary initial condition, yields an asymptotically vanishing system response. In Section 4, we extend the results of 
(Tokarzewski, 2002; 2006) by providing a general expression for output-zeroing inputs as well as a general form of the so-called zero dynamics for a particular case of the system (1) with uniform rank. Simple numerical examples are presented in Section 5.

Consider a system of the form (Górecki, et al., 1989; Richard, 2003)

$$
\begin{aligned}
& \dot{x}(t)=A x(t)+A_{1} x(t-h)+B u(t), \\
& y(t)=C x(t), \quad t \geq 0, \quad h>0,
\end{aligned}
$$

where $h$ is a known delay, for $x(\theta)=\varphi(\theta)$ for $\theta \in[-h, 0]$ and $x(t) \in \mathbb{R}^{n}, u(t) \in \mathbb{R}^{m}$ and $y(t) \in \mathbb{R}^{r} ; A, A_{1}, B, C$ are real matrices of appropriate dimensions. By $U$ we denote the set of admissible inputs which, for simplicity, consists of all continuous real-valued vector functions of time $u(\cdot):[0,+\infty) \rightarrow \mathbb{R}^{m}$. It is assumed that the initial condition $\varphi(\cdot):[-h, 0] \rightarrow \mathbb{R}^{n}$ is continuous or, more precisely, the set of initial conditions is the Banach space of continuous vector functions mapping the interval $[-h, 0]$ into $\mathbb{R}^{n}$ with the supremum norm

$$
\|\varphi\|_{\infty}=\max _{\theta \in[-h, 0]}\|\varphi(\theta)\|
$$

In other words, we consider this Banach space as a state space for the system (see, e.g., Richard, 2003).

Throughout this paper we use the Euclidean norm for vectors and the induced matrix norm for matrices, both denoted by $\|\cdot\|$. Recall (Hale, 1977) that for a given initial condition $\varphi(\cdot)$ and for a given input $u(\cdot) \in U$ by the solution of 1 we understand a continuous curve $x(t)=x(t, \varphi(\cdot), u(\cdot)), x(\cdot):[-h,+\infty) \rightarrow \mathbb{R}^{n}$, such that $x(\cdot)$ coincides with $\varphi(\cdot)$ on the interval $[-h, 0]$ and $x(\cdot)$ satisfies the first equation in (1) for all $t \geq 0$. Such a solution is unique.

The system (1) is asymptotically stable if and only if its characteristic equation $\operatorname{det}\left(s I-A-A_{1} e^{-s h}\right)=0$ has no roots with nonnegative real parts. As is known (Hale, 1977; Kharitonov, 1999; Kharitonov and Hinrichsen, 2004), if (1) is asymptotically stable, it is also exponentially stable, i.e., there exist positive constants $\alpha, \gamma$ such that for each solution $x(t, \varphi(\cdot))$ of the equation $\dot{x}(t)=A x(t)+A_{1} x(t-h)$ the inequality $\|x(t, \varphi(\cdot))\| \leq$ $\gamma\|\varphi\|_{\infty} e^{-\alpha t}$ holds for all $t \geq 0$.

Besides the above infinite-dimensional model, many classes of models have been proposed for the analysis of delay systems, e.g., models defined over the ring of polynomials (or the field of rational functions) in the delay operator $\nabla$, over the ring of rational causal transfer functions in $\nabla$, or over the ring of quasipolynomials (see (Richard, 2003) for an overview and (Kamen et al., 1985)).

\section{Invariant zeros and the output-zeroing problem}

Definition 1. A number $\lambda \in \mathbb{C}$ is an invariant zero of (1) if and only if there exist vectors $0 \neq x^{o} \in \mathbb{C}^{n}$ and $g \in \mathbb{C}^{m}$ such that

$$
P(\lambda)\left[\begin{array}{c}
x^{o} \\
g
\end{array}\right]=\left[\begin{array}{l}
0 \\
0
\end{array}\right],
$$

where

$$
P(s)=\left[\begin{array}{cc}
s I-A-A_{1} e^{-s h} & -B \\
C & 0
\end{array}\right] .
$$

By $Z^{I}$ we denote the set of invariant zeros of (1). The set $Z^{I}$ may be countable (empty, finite or infinite) or equal to the whole complex plane (i.e., $Z^{I}=\mathbb{C}$ ). In the latter case, the system (1) is called degenerate. Directly from Definition 1 it is clear that $Z^{I}$ is invariant under any change of coordinates $x^{\prime}=H x$. The point of departure for dynamical interpretation of invariant zeros is the following formulation of the output-zeroing problem (borrowed from (Isidori, 1995)): Find all pairs $\left(\varphi_{o}(\theta), u_{o}(t)\right)$ consisting of an admissible initial condition $\varphi_{o}():.[-h, 0] \rightarrow \mathbb{R}^{n}$ and an admissible input $u_{o}(t)$ such that the corresponding output of (1) is identically zero, i.e., $y(t)=0$ for all $t \geq 0$. Any nontrivial pair of this kind, i.e., such that $\varphi_{o}(\theta)$ and/or $u_{o}(t)$ is not identically zero, is called the output-zeroing input. In each outputzeroing input $\left(\varphi_{o}(\theta), u_{o}(t)\right), u_{o}(t)$ should be understood as an open-loop real-valued control signal which, when applied to (1) exactly under the initial condition $\varphi_{o}(\theta)$, yields $y(t)=0$ for all $t \geq 0$. The internal dynamics of (1) consistent with the constraint $y(t)=0$ for all $t \geq 0$ are called zero dynamics.

The set of all output-zeroing inputs for (1) complemented with the trivial pair $\left(\varphi_{o}(\theta) \equiv 0, u_{o}(t) \equiv 0\right)$ forms a linear space over $\mathbb{R}$. In fact, if $\left(\varphi_{o}^{1}(\theta), u_{o}^{1}(t)\right)$ and $\left(\varphi_{o}^{2}(\theta), u_{o}^{2}(t)\right)$ are output-zeroing inputs and give respectively solutions of the state equation $x_{o}^{1}(t)$ and $x_{o}^{2}(t)$, then, from the linearity of (1) and the uniqueness of solutions as well as from the fact that the set $U$ of admissible inputs forms a linear space over $\mathbb{R}$, it follows that each pair of the form $\left(\alpha \varphi_{o}^{1}(\theta)+\beta \varphi_{o}^{2}(\theta), \alpha u_{o}^{1}(t)+\beta u_{o}^{2}(t)\right)$, with arbitrarily fixed $\alpha, \beta \in \mathbb{R}$, is an output-zeroing input and yields the solution $\alpha x_{o}^{1}(t)+\beta x_{o}^{2}(t)$. In this space, we can distinguish a subspace consisting of all pairs of the form $\left(\varphi_{o}(\theta) \equiv 0, u_{o}(t)\right)$, where $u_{o}(t) \in \operatorname{ker} B$ for all $t \geq 0$. Each pair of this kind affects system equations in the same way as the trivial pair, i.e., it gives the identically zero solution and $y(t)=0$ for all $t \geq 0$. We do not associate this subspace with invariant zeros because it can exist independently of these zeros (cf. Example 1).

Lemma 1. If $\left(\varphi_{o}(\theta), u_{o}(t)\right)$ is an output-zeroing input for $(1)$ and $x_{o}(t)$ denotes the corresponding solution, then the input $u_{o}(t)$ when applied to the system under an arbitrary initial condition $\varphi(\theta)$ yields the solution of (1) of the 
form

$$
x\left(t, \varphi(\cdot), u_{o}(\cdot)\right)=x^{h}\left(t, \varphi(\cdot)-\varphi_{o}(\cdot)\right)+x_{o}(t),
$$

where $x^{h}\left(t, \varphi(\cdot)-\varphi_{o}(\cdot)\right)$ denotes the solution of the homogeneous (unforced) state equation in (1) corresponding to the initial condition $\varphi(\theta)-\varphi_{o}(\theta)$, and gives the system response

$$
y(t)=C x^{h}\left(t, \varphi(\cdot)-\varphi_{o}(\cdot)\right) .
$$

Proof. A simple proof that the right-hand side in (3) satisfies the initial condition $\varphi(\theta)$ and fulfills the state equation at $u(t)=u_{o}(t)$ follows by verification. Hence, the equality in (3) follows by the uniqueness of solutions. The equality in (4) follows by assumption (i.e., $C x_{o}(t)=0$ for $t \geq 0$ ).

Remark 1. In order to show that each invariant zero generates an output-zeroing input, it is convenient to treat the system (1) as a complex one, i.e., admitting complex valued initial conditions, inputs, solutions and outputs which are denoted respectively by $\tilde{\varphi}, \tilde{u}, \tilde{x}$ and $\tilde{y}$. Naturally, if $\tilde{x}(t)$ is a solution of (1) (treated as a complex system) corresponding to an input $\tilde{u}(t)$ and to an initial condition $\tilde{\varphi}(\theta)$, then its real part $\operatorname{Re} \tilde{x}(t)$ is a solution which corresponds to the initial condition $\operatorname{Re} \tilde{\varphi}(\theta)$ and to the input $\operatorname{Re} \tilde{u}(t)$. Analogously, $\operatorname{Im} \tilde{x}(t)$ (i.e., the imaginary part of $\tilde{x}(t))$ is a solution of (1) which corresponds to $\operatorname{Im} \tilde{\varphi}(\theta)$ and $\operatorname{Im} \tilde{u}(t)$. Furthermore, if a pair $(\tilde{\varphi}(\theta), \tilde{u}(t))$ is such that it gives also $\tilde{y}(t)=0$ for all $t \geq 0$, then the pairs $(\operatorname{Re} \tilde{\varphi}(\theta), \operatorname{Re} \tilde{u}(t))$ and $(\operatorname{Im} \tilde{\varphi}(\theta), \operatorname{Im} \tilde{u}(t))$ are output-zeroing inputs and give respectively the solutions $\operatorname{Re} \tilde{x}(t)$ and $\operatorname{Im} \tilde{x}(t)$.

\section{Geometric interpretation of invariant zeros}

As we show below (Lemma 3), Definition 1 clearly relates invariant zeros (even in the degenerate case) to the outputzeroing problem. To this end we first need the following.

Lemma 2. If $\lambda \in \mathbb{C}$ is an invariant zero of (1), i.e., a triple $\lambda, x^{o} \neq 0, g$ satisfies (2), then the input $\tilde{u}(t)=$ $g e^{\lambda t}$, when applied to (1) (treated as a complex system) under the initial condition $\tilde{\varphi}(\theta)=x^{o} e^{\lambda \theta}, \theta \in[-h, 0]$, yields the solution $\tilde{x}(t)=x^{o} e^{\lambda t}$ and the system response $\tilde{y}(t)=0$ for all $t \geq 0$.

Proof. By Definition 1, we have

$$
\lambda x^{o}-A x^{o}-A_{1} e^{-\lambda h} x^{o}=B g .
$$

Postmultiplying both sides of this equality by $e^{\lambda t}$, we obtain

$$
\dot{\tilde{x}}(t)-A \tilde{x}(t)-A_{1} \tilde{x}(t-h)=B \tilde{u}(t)
$$

for all $t \geq 0$. Moreover, from Definition 1 , we also have $C x^{o}=0$. Hence, $\tilde{y}(t)=C x^{o} e^{\lambda t}=0$ for all $t \geq 0$.
Lemma 3. Let $\lambda \in \mathbb{C}$ be an invariant zero of (1), i.e., let a triple $\lambda, x^{o} \neq 0$, g satisfy (2). Write $\lambda=\sigma+j \omega, x^{o}=$ $\operatorname{Re} x^{o}+j \operatorname{Im} x^{o}$ and $g=\operatorname{Re} g+j \operatorname{Im} g$. Then the pair $\left(\varphi_{o}(\theta), u_{o}(t)\right)$, where

$\varphi_{o}(\theta)=e^{\sigma \theta}\left(\operatorname{Re} x^{o} \cos \omega \theta-\operatorname{Im} x^{o} \sin \omega \theta\right), \quad \theta \in[-h, 0]$

and

$$
u_{o}(t)=e^{\sigma t}(\operatorname{Re} g \cos \omega t-\operatorname{Im} g \sin \omega t), \quad t \geq 0,
$$

is an output-zeroing input and yields the solution

$$
x_{o}(t)=e^{\sigma t}\left(\operatorname{Re} x^{o} \cos \omega t-\operatorname{Im} x^{o} \sin \omega t\right) .
$$

Similarly, the pair $\left(\varphi_{o}(\theta), u_{o}(t)\right)$, where

$$
\varphi_{o}(\theta)=e^{\sigma \theta}\left(\operatorname{Re} x^{o} \sin \omega \theta+\operatorname{Im} x^{o} \cos \omega \theta\right)
$$

and

$$
u_{o}(t)=e^{\sigma t}(\operatorname{Re} g \sin \omega t+\operatorname{Im} g \cos \omega t),
$$

is an output-zeroing input and yields the solution

$$
x_{o}(t)=e^{\sigma t}\left(\operatorname{Re} x^{o} \sin \omega t+\operatorname{Im} x^{o} \cos \omega t\right) .
$$

Proof. Of course, since in (1) all matrices are real, the complex conjugate of $\lambda$ is also an invariant zero, i.e., the triple $\bar{\lambda}=\sigma-j \omega, \bar{x}^{o}=\operatorname{Re} x^{o}-j \operatorname{Im} x^{o}$ and $\bar{g}=\operatorname{Re} g-$ $j \operatorname{Im} g$ satisfies (2). The proof of Lemma 3 follows easily from Lemma 2 and Remark 1.

The following result shows in particular that if the system (1) is asymptotically stable and a pair $\left(\varphi_{o}(\theta), u_{o}(t)\right)$ is an output-zeroing input, then the input signal $u_{o}(t)$, when applied to the system under an arbitrary initial condition $\varphi(\theta)$, yields an asymptotically vanishing system response, i.e., $y(t) \rightarrow 0$ as $t \rightarrow \infty$.

Lemma 4. Let $\left(\varphi_{o}(\theta), u_{o}(t)\right)$ be an output-zeroing input for an asymptotically stable system (1) and let $x_{o}(t)$ be the corresponding solution. By $x(t)$ denote a solution of (1) corresponding to $u_{o}(t)$ and to an arbitrary initial condition $\varphi(\theta)$. Then the Laplace transform of $x(t)$ can be written in the form

$$
\begin{aligned}
& X(s) \\
& =\left(s I-A-A_{1} e^{-s h}\right)^{-1}\left[\left(\varphi(0)-\varphi_{o}(0)\right)\right. \\
& \left.\quad+A_{1} e^{-s h} \int_{-h}^{0} e^{-s \theta}\left(\varphi(\theta)-\varphi_{o}(\theta)\right) \mathrm{d} \theta\right]+X_{o}(s),
\end{aligned}
$$

where

$$
X_{o}(s)=L\left(x_{o}(t)\right):=\int_{0}^{\infty} e^{-s t} x_{o}(t) \mathrm{d} t,
$$


while the Laplace transform of the corresponding system response, i.e., $y(t)=C x(t)$, can be written as

$$
\begin{aligned}
Y(s)= & C X(s) \\
= & C\left(s I-A-A_{1} e^{-s h}\right)^{-1}\left[\left(\varphi(0)-\varphi_{o}(0)\right)\right. \\
& \left.+A_{1} e^{-s h} \int_{-h}^{0} e^{-s \theta}\left(\varphi(\theta)-\varphi_{o}(\theta)\right) \mathrm{d} \theta\right] .
\end{aligned}
$$

Moreover, $y(t) \rightarrow 0$ as $t \rightarrow \infty$.

Proof. By assumption we have $\dot{x}_{o}(t)=A x_{o}(t)+$ $A_{1} x_{o}(t-h)+B u_{o}(t)$ for $t \geq 0$. Using the Laplace transformation for both sides of the above equality and taking into account that

$$
\begin{aligned}
& L\left(x_{o}(t-h)\right) \\
& \quad=\int_{0}^{\infty} e^{-s t} x_{o}(t-h) \mathrm{d} t \\
& \quad=\int_{0}^{h} e^{-s t} \varphi_{o}(t-h) \mathrm{d} t+\int_{h}^{\infty} e^{-s t} x_{o}(t-h) \mathrm{d} t \\
& \quad=e^{-s h} \int_{-h}^{0} e^{-s \theta} \varphi_{o}(\theta) \mathrm{d} \theta+e^{-s h} X_{o}(s),
\end{aligned}
$$

we obtain

$$
\begin{aligned}
X_{o}(s)= & \left(s I-A-A_{1} e^{-s h}\right)^{-1} \\
& \cdot\left[\varphi_{o}(0)+A_{1} e^{-s h} \int_{-h}^{0} e^{-s \theta} \varphi_{o}(\theta) \mathrm{d} \theta\right] \\
& +\left(s I-A-A_{1} e^{-s h}\right)^{-1} B U_{o}(s) .
\end{aligned}
$$

Analogously, for the Laplace transform of $x(t)$ we get

$$
\begin{aligned}
X(s)= & \left(s I-A-A_{1} e^{-s h}\right)^{-1} \\
& \cdot\left[\varphi(0)+A_{1} e^{-s h} \int_{-h}^{0} e^{-s \theta} \varphi(\theta) \mathrm{d} \theta\right] \\
& +\left(s I-A-A_{1} e^{-s h}\right)^{-1} B U_{o}(s) .
\end{aligned}
$$

Subtracting by sides (7) from (8), we get (5), i.e., (8) can be expressed as in (5). The relation (6) follows from (5) and $C X_{o}(s)=0$. The last claim of the lemma follows from Lemma 1 and the stability assumption (cf. Section 1). In fact, by virtue of (4), we can write

$$
\begin{aligned}
\|y(t)\| & =\left\|C x^{h}\left(t, \varphi(\cdot)-\varphi_{o}(\cdot)\right)\right\| \\
& \leq\|C\|\left\|x^{h}\left(t, \varphi(\cdot)-\varphi_{o}(\cdot)\right)\right\| \\
& \leq\|C\| \gamma\left\|\varphi-\varphi_{o}\right\|_{\infty} e^{-\alpha t} .
\end{aligned}
$$

\section{Zeros and the output-zeroing problem for systems of uniform rank}

In this section we consider a particular case of the system (1), namely, a square $m$-input $m$-output system of the form

$$
\dot{x}(t)=A_{1} x(t-h)+B u(t), \quad y(t)=C x(t), \quad t \geq 0
$$

of uniform rank, i.e., such that its first nonzero Markov parameter is nonsingular. We denote such a parameter by $C A_{1}^{k} B$, where $0 \leq k \leq n-1$, i.e., we assume $C B=$ $\cdots=C A_{1}^{k-1} B=0, C A_{1}^{k} B \neq 0$ and $\operatorname{rank} C A_{1}^{k} B=m$.

Remark 2. Recall that the transfer function matrix for (9) equals $C\left(s I-A_{1} e^{-s h}\right)^{-1} B$. In the half-plane $\operatorname{Re} s>\left\|A_{1}\right\|$ of the complex plane, it can be expanded in the power series

$$
\begin{aligned}
C\left(s I-A_{1} e^{-s h}\right)^{-1} B & \\
= & C B s^{-1}+C A_{1} B s^{-2} e^{-s h}+\ldots \\
& +C A_{1}^{k} B s^{-(k+1)} e^{-k s h}+\ldots
\end{aligned}
$$

By analogy to the standard case, the matrices $C A_{1}^{l} B$, $l=0,1,2, \ldots$ are called Markov parameters for the system (9).

Lemma 5. (Tokarzewski, 2006, p. 67) Define a matrix

$$
K_{k}:=I-B\left(C A_{1}^{k} B\right)^{-1} C A_{1}^{k} .
$$

Then $K_{k}$ has the following properties:

(i) $K_{k}^{2}=K_{k}$,

(ii) $\Sigma_{k}:=\left\{x: K_{k} x=x\right\}=\operatorname{ker}\left(C A_{1}^{k}\right)$, $\operatorname{dim} \Sigma_{k}=n-m$,

(iii) $\Omega_{k}:=\left\{x: K_{k} x=0\right\}=\operatorname{im} B, \quad \operatorname{dim} \Omega_{k}=m$,

(iv) $C^{n}\left(R^{n}\right)=\Sigma_{k} \oplus \Omega_{k}$.

Moreover,

(v) $K_{k} B=0, \quad C A_{1}^{k} K_{k}=0$,

(vi) $C\left(K_{k} A_{1}\right)^{l}= \begin{cases}C A_{1}^{l} & \text { for } \quad 0 \leq l \leq k, \\ 0 & \text { for } \quad l \geq k+1 .\end{cases}$

A general characterization of output-zeroing inputs and the corresponding solutions as well as zero dynamics for the system (9) is given in the following.

Theorem 1. A pair $\left(\varphi_{o}(\theta), u_{o}(t)\right)$ is an output-zeroing input for the system (9) of uniform rank if and only if

$$
\varphi_{o}(\theta) \in \bigcap_{l=1}^{k} \operatorname{ker} C A_{1}^{l}
$$

for each $\theta \in[-h, 0]$ and $\varphi_{o}(0) \in \operatorname{ker} C$, and $u_{o}(t)$ is such that its Laplace transform has the form

$$
\begin{aligned}
U_{o}(s) \\
=-\left(C A_{1}^{\mathrm{k}} B\right)^{-1} C A_{1}^{\mathrm{k}+1}\left[e^{-s h}\left(s I-K_{\mathrm{k}} A_{1} e^{-s h}\right)^{-1}\right. \\
\left.\quad \cdot\left\{\varphi_{o}(0)+s \int_{-h}^{0} e^{-s \theta} \varphi_{o}(\theta) \mathrm{d} \theta\right\}\right] .
\end{aligned}
$$


Moreover, if $x_{o}(t)$ is a solution corresponding to the output-zeroing input $\left(\varphi_{o}(\theta), u_{o}(t)\right)$, then its Laplace transform has the form

$$
\begin{aligned}
X_{o}(s)= & \left(s I-K_{\mathrm{k}} A_{1} e^{-s h}\right)^{-1}\left[\varphi_{o}(0)\right. \\
& \left.+K_{\mathrm{k}} A_{1} e^{-s h} \int_{-h}^{0} e^{-s \theta} \varphi_{o}(\theta) \mathrm{d} \theta\right]
\end{aligned}
$$

and $x_{o}(t)$ is entirely contained in $\bigcap_{l=0}^{k} \operatorname{ker} C A_{1}^{l}$, i.e., $x_{o}(t) \in \bigcap_{l=0}^{k} \operatorname{ker} C A_{1}^{l}$ for all $t \geq 0$.

Finally, the zero dynamics of the system have the form

$$
\dot{x}_{o}(t)=K_{k} A_{1} x_{o}(t-h)
$$

with the initial condition $\varphi_{o}(\theta), \theta \in[-h, 0]$.

Proof. For the proof of necessity, let us suppose that $\left(\varphi_{o}(\theta), u_{o}(t)\right)$ is an output-zeroing input and $x_{o}(t)$ is the corresponding solution. Thus we have $y(t)=C x_{o}(t)=0$ for all $t \geq 0$ and, consequently, $C \varphi_{o}(0)=0$. Moreover, since $\dot{y}(t)=C \dot{x}_{o}(t)=C A_{1} x_{o}(t-h)+C B u_{o}(t)$ and $\dot{y}(t) \equiv 0$ as well as $C B=0$, we get $C A_{1} x_{o}(t-h)=0$ for all $t \geq 0$. This last equality yields $C A_{1} \varphi_{o}(\theta)=0$ for $\theta \in[-h, 0]$ and $C A_{1} x_{o}(t)=0$ for $t \geq 0$. Next, we have $0=C A_{1} \dot{x}_{o}(t)=C A_{1}^{2} x_{o}(t-h)+C A_{1} B u_{o}(t)$ and, since $C A_{1} B=0$, we obtain $C A_{1}^{2} x_{o}(t-h)=0$ for $t \geq 0$. Consequently, $C A_{1}^{2} \varphi_{o}(\theta)=0$ for $\theta \in[-h, 0]$ and $C A_{1}^{2} x_{o}(t)=0$ for $t \geq 0$. Proceeding analogously, we obtain, after a finite number of steps, the following relations:

$$
\begin{aligned}
& \varphi_{o}(\theta) \in \bigcap_{l=1}^{k} \operatorname{ker} C A_{1}^{l} \text { for } \theta \in[-h, 0], \\
& \varphi_{o}(0) \in \bigcap_{l=0}^{k} \operatorname{ker} C A_{1}^{l}, \\
& x_{o}(t) \in \bigcap_{l=0}^{k} \operatorname{ker} C A_{1}^{l}, \quad \dot{x}_{o}(t) \in \bigcap_{l=0}^{k} \operatorname{ker} C A_{1}^{l} \text { for } t \geq 0 .
\end{aligned}
$$

From (15) and from Lemma 5 (see (10)), we obtain

$$
\begin{aligned}
K_{k} x_{o}(t) & =x_{o}(t), \\
K_{k} \dot{x}_{o}(t) & =\dot{x}_{o}(t), \\
K_{k} \varphi_{o}(\theta) & =\varphi_{o}(\theta) .
\end{aligned}
$$

In the last step, we can write

$$
0=C A_{1}^{k} \dot{x}_{o}(t)=C A_{1}^{k+1} x_{o}(t-h)+C A_{1}^{k} B u_{o}(t),
$$

which yields

$$
u_{o}(t)=-\left(C A_{1}^{k} B\right)^{-1} C A_{1}^{k+1} x_{o}(t-h) .
$$

On the other hand, premultiplying both sides of the equality $\dot{x}_{o}(t)=A_{1} x_{o}(t-h)+B u_{o}(t)$ by $K_{k}$ and taking into account (15) and Lemma 5(v), we get the relation

$$
\dot{x}_{o}(t)=K_{k} A_{1} x_{o}(t-h) \quad \text { with } \varphi_{o}(\theta), \theta \in[-h, 0] \text {, }
$$

which represents the zero dynamics of the system. Thus, $u_{o}(t)$ in (17) is determined by $x_{o}(t-h)$, where $x_{o}(t-h)$ follows from the solution of (18) under the initial condition $\varphi_{o}(\theta)$. Taking the Laplace transform of both sides of (18), we obtain $X_{o}(s)$ as in (13). Finally, we shall show that $u_{o}(t)$ (or, more precisely, its Laplace transform) can be determined merely by the initial condition. To this end, we take the Laplace transform of both sides of (17) and, after simple calculations, we get $U_{o}(s)$ in the form (12). This ends the proof of necessity.

For the proof of sufficiency, we assume that $\varphi_{o}(\theta)$ satisfies the conditions (11) and $u_{o}(t)$ is such that its Laplace transform has the form (12). We are to show that $\left(\varphi_{o}(\theta), u_{o}(t)\right)$ is an output-zeroing input and the corresponding solution has the Laplace transform as in (13). To this end, we first search for a solution $x(t)$ of (9) corresponding to $\varphi_{o}(\theta)$ and to $U_{o}(s)$ as in (12). Thus, for the Laplace transform of this solution, we can write

$$
\begin{aligned}
X(s)= & \left(s I-A_{1} e^{-s h}\right)^{-1} \\
& \cdot\left[\varphi_{o}(0)+A_{1} e^{-s h} \int_{-h}^{0} e^{-s \theta} \varphi_{o}(\theta) \mathrm{d} \theta\right] \\
& +\left(s I-A_{1} e^{-s h}\right)^{-1} B U_{o}(s) .
\end{aligned}
$$

Substituting (12) into (19) and using the definition of $K_{k}$ (see (10)) as well as the identities

$$
\begin{aligned}
& I-B\left(C A_{1}^{k} B\right)^{-1} C A_{1}^{k+1} e^{-s h}\left(s I-K_{k} A_{1} e^{-s h}\right)^{-1} \\
& \quad=\left(s I-A_{1} e^{-s h}\right)\left(s I-K_{k} A_{1} e^{-s h}\right)^{-1}, \quad(20) \\
& \quad A_{1} e^{-s h} \\
& \quad-B\left(C A_{1}^{k} B\right)^{-1} C A_{1}^{k+1} s e^{-s h}\left(s I-K_{k} A_{1} e^{-s h}\right)^{-1} \\
& \quad=\left(s I-A_{1} e^{-s h}\right) K_{k} A_{1} e^{-s h}\left(s I-K_{k} A_{1} e^{-s h}\right)^{-1},
\end{aligned}
$$

we transform the right-hand side of (19) into the form (13), i.e., we have obtained $X(s)=X_{o}(s)$.

Now, we shall show that $X_{o}(s) \in \bigcap_{l=0}^{k} \operatorname{ker} C A_{1}^{l}$. To this end, the above obtained solution $X_{o}(s)$ (13) is expressed in the form of the following identity:

$$
\begin{aligned}
s X_{o}(s) & -K_{\mathrm{k}} A_{1} e^{-s h} X_{o}(s) \\
= & \varphi_{o}(0)+K_{\mathrm{k}} A_{1} e^{-s h} \int_{-h}^{0} e^{-s \theta} \varphi_{o}(\theta) \mathrm{d} \theta .
\end{aligned}
$$

Then, premultiplying both sides of (22) subsequently by $C\left(K_{k} A_{1}\right)^{k}, C\left(K_{k} A_{1}\right)^{k-1}, \ldots, C\left(K_{k} A_{1}\right), C$ and using $C B=\cdots=C A_{1}^{k-1} B=0$, (11) and Lemma 5(vi), 
we obtain $C A_{1}^{k} X_{o}(s)=0, C A_{1}^{k-1} X_{o}(s)=0, \ldots$, $C A_{1} X_{o}(s)=0, C X_{o}(s)=0$, i.e., we have the relation $X_{o}(s) \in \bigcap_{l=0}^{k} \operatorname{ker} C A_{1}^{l}$. This means that $x_{o}(t) \in$ $\bigcap_{l=0}^{k} \operatorname{ker} C A_{1}^{l}$. Since, in particular, $x_{o}(t) \in \operatorname{ker} C$, we infer that $\left(\varphi_{o}(\theta), u_{o}(t)\right)$ is an output-zeroing input.

In the remaining part of this section we characterize invariant zeros as the roots of some quasi-polynomial. To this end we first need the following result.

Lemma 6. A number $\lambda \in \mathbb{C}$ is an invariant zero of the system (9) with uniform rank if and only if there exists a vector $0 \neq x^{o} \in \mathbb{C}^{n}$ such that

$$
\lambda x^{o}-K_{k} A_{1} e^{-\lambda h} x^{o}=0, \quad C x^{o}=0 .
$$

Proof. If $\lambda \in \mathbb{C}$ is an invariant zero, then, by Definition 1 , there exist $0 \neq x^{o} \in \mathbb{C}^{n}$ and $g \in \mathbb{C}^{m}$ such that $\lambda x^{o}-A_{1} e^{-\lambda h} x^{O}=B g, C x^{O}=0$. Premultiplying successively the first equality by $C, C A_{1}, \ldots, C A_{1}^{k-1}$ and taking into account $C B=\ldots=C A_{1}^{k-1} B=0$ and $C x^{o}=0$, we get $x_{o} \in \bigcap_{l=0}^{k} \operatorname{ker} C A_{1}^{l}$, i.e., $x^{o} \in \Sigma_{k}$ (see Lemma 5(ii)) and, consequently, $K_{k} x^{o}=x^{o}$. Now, premultiplying the equality $\lambda x^{o}-A_{1} e^{-\lambda h} x^{o}=B g$ by $K_{k}$ and using Lemma $5(\mathrm{v})$, we get the first equality in (23). Conversely, if (23) holds, then, using the definition of $K_{k}$ (10) and taking $g=-\left(C A_{1}^{k} B\right)^{-1} C A_{1}^{k+1} e^{-\lambda h} x^{o}$, we can write the first equality in (23) as $\lambda x^{o}-A_{1} e^{-\lambda h} x^{o}=$ $B g$.

With the system (9) of uniform rank we associate the triple of matrices $\left(K_{k} A_{1}, B, C\right)$. Consider the pair of matrices $\left(K_{k} A_{1}, C\right)$. As is known (Tokarzewski, 2006, p. 140), the observability matrix for $\left(K_{k} A_{1}, C\right)$ has the rank $m(k+1)$, i.e.,

$$
\operatorname{rank}\left[\begin{array}{c}
C \\
C\left(K_{k} A_{1}\right) \\
\left.\cdot \dot{A_{1}}\right)^{n-1}
\end{array}\right]=m(k+1) .
$$

To the triple $\left(K_{k} A_{1}, B, C\right)$ we can apply a decomposition $(\bar{o} / o)$ into an unobservable $\bar{o}$ and an observable $(o)$ part. Let $x^{\prime}=H x$ denote a change of coordinates which leads to the $(\bar{o} / o)$ decomposition with the matrices

$$
\begin{aligned}
\left(K_{k} A_{1}\right)^{\prime} & =\left[\begin{array}{cc}
\left(K_{k} A_{1}\right)_{\bar{o}}^{\prime} & \left(K_{k} A_{1}\right)_{1,2}^{\prime} \\
0 & \left(K_{k} A_{1}\right)_{o}^{\prime}
\end{array}\right], \\
B^{\prime} & =\left[\begin{array}{c}
B_{\bar{o}}^{\prime} \\
B_{o}^{\prime}
\end{array}\right], \\
C^{\prime} & =\left[\begin{array}{ll}
0 & C_{o}^{\prime}
\end{array}\right], \\
x^{\prime} & =\left[\begin{array}{l}
x_{\bar{o}}^{\prime} \\
x_{o}^{\prime}
\end{array}\right], \quad \operatorname{dim} x_{\bar{o}}^{\prime}=n-m(k+1),
\end{aligned}
$$

where the pair $\left(\left(K_{k} A_{1}\right)_{o}^{\prime}, C_{o}^{\prime}\right)$ is observable, i.e., its observability matrix has the rank $m(k+1)$. Now, with the notation used above, we can formulate the following result.
Theorem 2. Consider the system $S\left(A_{1} e^{-s h}, B, C\right)$ (9) with uniform rank. Then a number $\lambda \in \mathbb{C}$ is an invariant zero of the system if and only if $\lambda$ is a root of the equation $\operatorname{det}\left(s I_{\bar{o}}-\left(K_{k} A_{1}\right)_{\bar{o}}^{\prime} e^{-s h}\right)=0$.

Proof. With the system $S\left(A_{1} e^{-s h}, B, C\right)$ (9) of uniform rank we associate an auxiliary closed-loop state feedback system $S\left(K_{k} A_{1} e^{-s h}, B, C\right)$ obtained from (9) by introducing the control law

$$
u(t)=v(t)+F x(t-h)
$$

where the state-feedback matrix equals

$$
F=-\left(C A_{1}^{k} B\right)^{-1} C A_{1}^{k+1} .
$$

To $S\left(K_{k} A_{1} e^{-s h}, B, C\right)$ we apply a change of coordinates which leads to the decomposition (24). The system obtained in this way is denoted as $S\left(\left(K_{k} A_{1}\right)^{\prime} e^{-s h}, B^{\prime}, C^{\prime}\right)$. Moreover, consider the system $S\left(A_{1}^{\prime} e^{-s h}, B^{\prime}, C^{\prime}\right)$ obtained from $S\left(A_{1} e^{-s h}, B, C\right)$ by the same change of coordinates. Note that forming for $S\left(A_{1}^{\prime} e^{-s h}, B^{\prime}, C^{\prime}\right)$ the auxiliary closed-loop state feedback system of the form $S\left(K_{k}^{\prime} A_{1}^{\prime} e^{-s h}, B^{\prime}, C^{\prime}\right)$, where, by definition, $K_{k}^{\prime}:=I-B^{\prime}\left(C^{\prime}\left(A_{1}^{\prime}\right)^{k} B^{\prime}\right)^{-1} C^{\prime}\left(A_{1}^{\prime}\right)^{k}$, we obtain $S\left(\left(K_{k} A_{1}\right)^{\prime} e^{-s h}, B^{\prime}, C^{\prime}\right)$. This fact follows from the relation $\left(K_{k} A_{1}\right)^{\prime}=K_{k}^{\prime} A_{1}^{\prime}$ (Tokarzewski, 2006, p. 142). Of course, the set of invariant zeros of $S\left(A_{1}^{\prime} e^{-s h}, B^{\prime}, C^{\prime}\right)$ is the same as that of $S\left(A_{1} e^{-s h}, B, C\right)$. Moreover, to the systems $S\left(A_{1}^{\prime} e^{-s h}, B^{\prime}, C^{\prime}\right)$ and $S\left(K_{k}^{\prime} A_{1}^{\prime} e^{-s h}, B^{\prime}, C^{\prime}\right)$ (or, which is the same, to $S\left(\left(K_{k} A_{1}\right)^{\prime} e^{-s h}, B^{\prime}, C^{\prime}\right)(24)$ ) we can apply Lemma 6, i.e., a number $\lambda \in \mathbb{C}$ is an invariant zero of $S\left(A_{1}^{\prime} e^{-s h}, B^{\prime}, C^{\prime}\right)$ if and only if there exists a vector $0 \neq x^{\prime o} \in \mathbb{C}^{n}$ such that $\lambda x^{\prime o}-\left(K_{k} A_{1}\right)^{\prime} e^{-\lambda h} x^{\prime o}=$ 0 and $C^{\prime} x^{\prime o}=0$. Now, for the proof of Theorem 2, we only need to show that a number $\lambda$ is a root of the equation $\operatorname{det}\left(s I_{\bar{o}}-\left(K_{k} A_{1}\right)_{\bar{o}}^{\prime} e^{-s h}\right)=0$ if and only if there exists a vector $0 \neq x^{\prime o} \in \mathbb{C}^{n}$ such that $\lambda x^{\prime o}-\left(K_{k} A_{1}\right)^{\prime} e^{-\lambda h} x^{\prime o}=$ 0 and $C^{\prime} x^{\prime o}=0$. Using (24), the last two relations can be written as

$$
\begin{aligned}
& \lambda x_{\bar{o}}^{\prime o}-\left(K_{k} A_{1}\right)_{\bar{o}}^{\prime} e^{-\lambda h} x_{\bar{o}}^{{ }^{o}}-\left(K_{k} A_{1}\right)_{12}^{\prime} e^{-\lambda h} x_{o}^{\prime o}=0, \\
& \lambda x_{o}^{\prime o}-\left(K_{k} A_{1}\right)_{o}^{\prime} e^{-\lambda h} x_{o}^{\prime o}=0, . \\
& C_{o}^{\prime} x_{o}^{\prime o}=0
\end{aligned}
$$

Suppose now that

$$
\operatorname{det}\left(\lambda I_{\bar{o}}-\left(K_{k} A_{1}\right)_{\bar{o}}^{\prime} e^{-\lambda h}\right)=0 .
$$

Then there exists an $x_{\bar{o}}^{\prime o} \neq 0$ such that $\left(\lambda I_{\bar{o}}\right.$ $\left.\left(K_{k} A_{1}\right)_{\bar{o}}^{\prime} e^{-\lambda h}\right) x_{\bar{o}}^{\prime o}=0$. Of course, $(25)$ will be satisfied for

$$
x^{\prime o}=\left[\begin{array}{c}
x_{\bar{o}}^{\prime o} \\
0
\end{array}\right] \text {. }
$$


In order to prove the converse, suppose that (25) is satisfied and $\lambda$ is not a root of the equation $\operatorname{det}\left(s I_{\bar{o}}-\right.$ $\left.\left(K_{k} A_{1}\right)_{\bar{o}}^{\prime} e^{-s h}\right)=0$. Then, however, $\lambda$ must be a root of $\operatorname{det}\left(s I_{o}-\left(K_{k} A_{1}\right)_{o}^{\prime} e^{-s h}\right)=0$. We shall discuss separately two cases. In the first one, suppose that in (25) there is $x_{o}^{\prime o} \neq 0$, i.e., $\left(\lambda I_{o}-\left(K_{k} A_{1}\right)_{o}^{\prime} e^{-\lambda h}\right) x_{o}^{\prime o}=0$, $C_{o}^{\prime} x_{o}^{\prime o}=0$. Since the pair $\left(\left(K_{k} A_{1}\right)_{o}^{\prime}, C_{o}^{\prime}\right)$ is observable, i.e., its observability matrix has full column rank $(m(k+1))$, it is easy to show by reductio ad absurdum that the pair $\left(\left(K_{k} A_{1}\right)_{o}^{\prime} e^{-s h}, C_{o}^{\prime}\right)$ is spectrally observable (Lee and Olbrot, 1981), i.e.,

$$
\operatorname{rank}\left[\begin{array}{c}
s I_{o}-\left(K_{k} A_{1}\right)_{o}^{\prime} e^{-s h} \\
C_{o}^{\prime}
\end{array}\right]=m(k+1)
$$

for each $s \in \mathbb{C}$.

This yields, however, the contradiction $x_{o}^{\prime o}=0$. In the second case, suppose that in (25) is $x_{o}^{\prime o}=0$. Then, we must have $x_{\bar{o}}^{\prime} \neq 0$, which contradicts the assumption that $\lambda$ is not a root of the equation $\operatorname{det}\left(s I_{\bar{o}}-\left(K_{k} A_{1}\right)_{\bar{o}}^{\prime} e^{-s h}\right)=$ 0 .

\section{Examples}

Example 1. Consider the system (1) with the matrices

$$
\begin{aligned}
A & =A_{1}=\left[\begin{array}{cc}
-1 & 0 \\
0 & -2
\end{array}\right], \\
B & =\left[\begin{array}{ccc}
-2 & 1 & 0 \\
0 & 0 & 1
\end{array}\right], \\
C & =\left[\begin{array}{ll}
1 & 0 \\
0 & 1
\end{array}\right] .
\end{aligned}
$$

This system has no invariant zeros since the matrix $C$ is nonsingular and for this reason Definition 1 cannot be satisfied for any triple $\lambda, x^{o} \neq 0, g$. On the other hand, output-zeroing inputs of the form $\left(\varphi_{o}(\theta) \equiv 0, u_{o}(t)\right)$, where $u_{o}(t) \in \operatorname{ker} B$ for all $t \geq 0$, exist.

Example 2. The following result characterizes the invariant zeros of a certain class of systems of the form (1). If in a square system (1) (i.e., $m=r$ ) the matrix $B$ has full column rank, then

(a) $\lambda \in Z^{I}$ if and only if $\operatorname{det} P(\lambda)=0$;

(b) the system (1) is degenerate if and only if

$$
\operatorname{det} P(s) \equiv 0,
$$

where

$$
P(s)=\left[\begin{array}{cc}
s I-A-A_{1} e^{-s h} & -B \\
C & 0
\end{array}\right] .
$$

The proof of this result is completely analogous to that given in (Tokarzewski, 2006, p. 55).
Example 3. In a system of the form (9), let

$$
\begin{aligned}
A_{1} & =\left[\begin{array}{ccc}
0 & 1 & 0 \\
0 & 0 & 1 \\
-1 & -2 & -1
\end{array}\right], \\
B & =\left[\begin{array}{ll}
0 & 0 \\
0 & 1 \\
1 & 0
\end{array}\right], \\
C & =\left[\begin{array}{ccc}
-2 & -1 & 0 \\
0 & 1 & 0
\end{array}\right] .
\end{aligned}
$$

As follows from Example 2, this system is degenerate since $\operatorname{det} P(s) \equiv 0$.

Example 4. In a system of the form (9), let

$$
\begin{aligned}
A_{1} & =\left[\begin{array}{llll}
0 & 0 & 0 & 0 \\
0 & 0 & 1 & 0 \\
0 & 0 & 0 & 1 \\
0 & 0 & 0 & 0
\end{array}\right], \\
B & =\left[\begin{array}{ll}
1 & 0 \\
0 & 0 \\
0 & 0 \\
0 & 1
\end{array}\right], \\
C & =\left[\begin{array}{llll}
1 & 0 & 0 & 0 \\
0 & 0 & 1 & 0
\end{array}\right] .
\end{aligned}
$$

Since $\operatorname{det} P(s)=s e^{-s h}$, by virtue of Example 2, the system has exactly one single invariant zero $\lambda=0$.

Example 5. Consider a system (9) of uniform rank with the matrices

$$
\begin{aligned}
A_{1} & =\left[\begin{array}{ccc}
-1 & 1 & 1 \\
0 & -1 & 1 \\
0 & 0 & -1
\end{array}\right], \\
B & =\left[\begin{array}{ll}
0 & 0 \\
0 & 1 \\
1 & 0
\end{array}\right], \\
C & =\left[\begin{array}{lll}
0 & 0 & 1 \\
1 & 1 & 0
\end{array}\right] .
\end{aligned}
$$

Using Theorem 2, we first find the invariant zeros of the system. The first nonzero Markov parameter is $C B$, hence, according to Lemma 5, $k=0$ and

$$
\begin{aligned}
K_{0} & =\left[\begin{array}{ccc}
1 & 0 & 0 \\
-1 & 0 & 0 \\
0 & 0 & 0
\end{array}\right], \\
K_{0} A_{1} & =\left[\begin{array}{ccc}
-1 & 1 & 1 \\
1 & -1 & -1 \\
0 & 0 & 0
\end{array}\right]
\end{aligned}
$$

In order to obtain an $(\bar{o} / o)$ decomposition of the triple $\left(K_{o} A_{1}, B, C\right)$, we take the change of coordinates 
$x^{\prime}=H x$, where

$$
H=\left[\begin{array}{lll}
1 & 0 & 0 \\
0 & 0 & 1 \\
1 & 1 & 0
\end{array}\right]
$$

Then

$$
\left(K_{0} A_{1}\right)^{\prime}=\left[\begin{array}{ccc}
-2 & 1 & 1 \\
0 & 0 & 0 \\
0 & 0 & 0
\end{array}\right],
$$

where $\left(K_{0} A_{1}\right)_{\bar{o}}^{\prime}=-2$. Thus the invariant zeros of the system are the roots of the equation $s+2 e^{-s h}=0$.

Of course, it is easy to verify (see, e.g., (Hale, 1977, Theorem A5)) that the system is stable if and only if $0<$ $h<\pi / 2$, whereas all its invariant zeros remain in $\mathbb{C}^{-}=$ $\{s \in \mathbb{C}: \operatorname{Re} s<0\}$ if and only if $0<h<\pi / 4$. Finally, in the new coordinates, the zero dynamics of the system have the form (Theorem 1)

$$
\dot{x}^{\prime}(t)=\left(K_{0} A_{1}\right)^{\prime} x^{\prime}(t-h)
$$

where

$$
x^{\prime}=\left[\begin{array}{l}
x_{1}^{\prime} \\
x_{2}^{\prime} \\
x_{3}^{\prime}
\end{array}\right]
$$

i.e.,

$$
\begin{aligned}
& \dot{x}_{1}^{\prime}(t)=-2 x_{1}^{\prime}(t-h)+x_{2}^{\prime}(t-h)+x_{3}^{\prime}(t-h), \\
& \dot{x}_{2}^{\prime}(t)=0 \\
& \dot{x}_{3}^{\prime}(t)=0
\end{aligned}
$$

where the initial condition $\varphi^{\prime}(\theta), \theta \in[-h, 0]$, must satisfy the condition (see (11))

$$
\varphi^{\prime}(0) \in \operatorname{ker} C^{\prime},
$$

where

$$
C^{\prime}=\left[\begin{array}{lll}
0 & 1 & 0 \\
0 & 0 & 1
\end{array}\right]
$$

\section{Concluding remarks}

In this paper we introduced the concept of invariant zeros for an LTI system with time delay in state (Definition 1). The problem of zeroing the system output as well as the output zeroing inputs are defined. The relationship between invariant zeros and the output-zeroing problem was presented (Lemmas 2 and 3). It was also shown that for an asymptotically stable system (1) the output-zeroing control signal, when applied to the system under an arbitrary initial condition, yields an asymptotically vanishing system response (Lemma 4). For systems with uniform rank, a necessary and sufficient condition for outputzeroing inputs was formulated (Theorem 1). Finally, it was shown that for such systems invariant zeros can be characterized as the roots of a certain quasi-polynomial (Theorem 2). Further research concerning invariant zeros and the output-zeroing problem can be focused on extending the obtained results to rectangular systems of the form (9) by using the Moore-Penrose pseudo-inverse and/or singular value decomposition for the first nonzero Markov parameter. Systems of the form (1) can be analyzed in this way assuming $C B \neq 0$.

\section{References}

Bourles, H. and Fliess, M. (1997). Finite poles and zeros of linear systems: An intrinsic approach, International Journal of Control 68(4):897-922.

Górecki, H., Fuksa, S., Grabowski, P. and Korytowski, A. (1989). Analysis and Synthesis of Time Delay Systems, PWN/Wiley, Warsaw/Chichester.

Hale, J. (1977). Theory of Functional Differential Equations, Springer, New York, NY.

Isidori, A. (1995). Nonlinear Control Systems, Springer Verlag, London.

Kamen, E. W., Khargonekar, P. P. and Tannenbaum, A. (1985). Stabilization of time-delay systems using finitedimensional compensators, IEEE Transactions on Automatic Control 30(1): 75-78.

Kharitonov, V. L. (1999). Robust stability analysis of time delay systems: A survey, Annual Reviews in Control 23(1): 185196.

Kharitonov V. L. and Hinrichsen, D. (2004). Exponential estimates for time delay systems, Systems and Control Letters 53(5):395-405.

Lee, E. B. and Olbrot, A. W. (1981). Observability and related structural results for linear hereditary systems, International Journal of Control 34(6):1061-1078.

MacFarlane, A. G. J. and Karcanias, N. (1976). Poles and zeros of linear multivariable systems: A survey of the algebraic, geometric and complex variable theory, International Journal of Control 24(1):33-74.

Marro, G. (1996). Multivariable regulation in geometric terms: Old and new results, in C. Bonivento, G. Marro, R. Zanasi (Eds.), Colloquium on Automatic Control, Lecture Notes in Control and Information Sciences, Vol. 215, Springer Verlag, London, pp. 77-138.

Pandolfi, L. (1982). Transmission zeros of systems with delays, International Journal of Control 36(6): 959-976.

Pandolfi, L. (1986). Disturbance decoupling and invariant subspaces for delay systems, Applied Mathematics and Optimization 14(1): 55-72.

Richard, J. P. (2003). Time-delay systems: An overview of some recent advances and open problems, Automatica 39(10): 1667-1694.

Schrader, C. B. and Sain, M. K. (1989). Research on system zeros: A survey, International Journal of Control 50(4):1407-1433. 
Sontag, E. D. (1990). Mathematical Control Theory, Springer Verlag, New York, NY.

Tokarzewski, J. (2002). Zeros in Linear Systems: A Geometric Approach, Warsaw University of Technology Press, Warsaw.

Tokarzewski, J. (2006). Finite Zeros in Discrete-Time Control Systems, Lecture Notes in Control and Information Sciences, Vol. 338, Springer Verlag, Berlin.

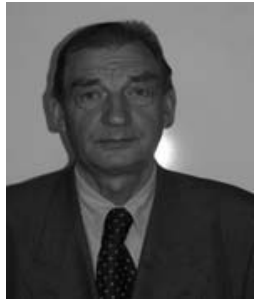

Jerzy Tokarzewski received the Ph.D. degree in 1975 and the D.Sc. (habilitation) degree in 1986 from the Warsaw University of Technology, both in electrical engineering. He is currently a professor at the Department of Electrical Engineering, Warsaw University of Technology. He is the author of Zeros in Linear Systems: A Geometric Approach (Warsaw University of Technology Press, 2002) and Finite Zeros in Discrete-Time Control Systems (Springer Verlag, 2006). His current research interests include fractional order control systems and systems with delay.

Received: 10 August 2008

Revised: 7 January 2009 